\title{
Germanica
}

\section{Lebenswege und Zeiterfahrung in Uwe Timms Der Freund und der Fremde}

Itinéraires personnels et expérience du temps dans L'Ami et l'étranger de Uwe Timm

Personal ways and time experience in Uwe Timm's "The Friend and the Stranger"

\section{Gert Sautermeister}

\section{OpenEdition}

\section{Journals}

Édition électronique

URL : https://journals.openedition.org/germanica/260

DOI : 10.4000/germanica.260

ISSN : 2107-0784

\section{Éditeur}

Université de Lille

\section{Édition imprimée}

Date de publication : 1 juin 2007

Pagination : 75-90

ISBN : 2-913857-19-1

ISSN : 0984-2632

\section{Référence électronique}

Gert Sautermeister, "Lebenswege und Zeiterfahrung in Uwe Timms Der Freund und der Fremde“,

Germanica [Online], 40 | 2007, Online erschienen am: 16 Februar 2010, abgerufen am 21 September 2021. URL: http://journals.openedition.org/germanica/260 ; DOI: https://doi.org/10.4000/germanica. 260

Ce document a été généré automatiquement le 21 septembre 2021.

(c) Tous droits réservés 


\section{Lebenswege und Zeiterfahrung in Uwe Timms Der Freund und der Fremde}

Itinéraires personnels et expérience du temps dans L'Ami et l'étranger de Uwe Timm

Personal ways and time experience in Uwe Timm's "The Friend and the Stranger"

Gert Sautermeister

Die Seele, der im Leben ihr göttlich Recht Nicht ward, sie ruht auch drunten im Orkus nicht.

(Friedrich Hölderlin, An die Parzen)

1 In Anlehnung an den Titel dieses Heftes habe ich für die Überschriften meiner folgenden Kapitel meist topographische Bezeichnungen gewählt. Einige davon verleugnen keineswegs - nach dem Beispiel Anderswo - ihren metaphorischen Charakter.

\section{Tatort (1)}

2 Der Ich-Erzähler - er repräsentiert den Autor selbst - erinnert sich zu Beginn seiner Geschichte an seinen Studienaufenthalt in Paris. Im Juni 1967 hatte ihn eine bestürzende Nachricht ereilt. Sie betraf einen Freund in Deutschland. Wir lesen :

Einige Tage danach sah ich sein Foto in einer Zeitschrift, und dieses Wiedersehen war wie ein Schock. [...] Er liegt auf dem Asphalt, bekleidet mit einer Khakihose, einem langärmeligen Hemd, der Arm ausgestreckt, die Hand entspannt geöffnet, die Augen geschlossen, als schliefe er. Neben ihm kniet eine junge Frau in einem schwarzen Kleid oder Umhang. [...] Sie blickt nach oben, so als wolle sie etwas fragen oder eine Anweisung geben, und hält, eine zärtliche Geste, seinen Kopf im Nacken. Deutlich ist das Blut am Kopf und am Boden zu sehen. [...] Ich saß in der Bibliothek über die Zeitschrift gebeugt und sah ihn, und in dem Moment wurde aus 
dem abstrakten Wissen um den Verlust eine körperlich spürbare Empfindung - ein Schmerz [...].

Wäre er infolge einer Krankheit oder eines Unfalls gestorben, wäre Trauer um ihn möglich gewesen, so aber war sein Tod ein Skandal, der in Kommentaren, Erklärungen und Gegenerklärungen abgehandelt wurde. [...] Das Sensationelle seines Todes verhinderte in den ersten Wochen und Monaten ein einfühlsames Erinnern. [...] Es blieb aber der Vorsatz, mehr noch, die Verpflichtung, über ihn zu schreiben. Ein Erzählen, das nur gelingen konnte - und diese Einsicht mußte erst wachsen -, wenn ich auch über mich erzählte. (S. 11ff.) ${ }^{1}$

3 Die Rede ist vom Tod des Freundes Benno Ohnesorg am 2. Juni 1967 und seinen Folgen. Dieser gewaltsam herbeigeführte Tod verwandelte in der Bundesrepublik « den schon vorhandenen, aufgestauten Unwillen » über die gesellschaftlichen Verhältnisse "in den Willen zur Tat» (S. 118) : zur Formierung der deutschen Protestbewegung. Den Erzähler verstört das höhnische, wahrhaft absurde Mißverhältnis zwischen der brutalen Polizeigewalt, die im Totschlag gipfelte, und dem gewaltlosen, feinfühligen Wesen des Freundes. Daß «der Mensch dem Menschen ein Helfer » sei - diese Maxime Bert Brechts hatte seine eigene Ethik bestimmt (vgl.S. 19). Krass bis zur Absurdität stach dagegen sein Schicksal ab : die Vernichtung seines sechsundzwanzig Jahre jungen Lebens. Dieses Leben in die erzählte Gegenwart zu retten, ist die Absicht des Schreibenden. Er entwirft nach Maßgabe seiner zweijährigen Freundschaft mit Benno Ohnesorg und aller ihn betreffenden Zeugnisse, schriftlichen und mündlichen, ein Charakterbild, so authentisch wie nur möglich. Und ein Epochenbild gleichzeitig. Beides begleitet von einem Selbstporträt, das aus dem Gang des eigenen Lebens entsteht. Eine Trias bewegender Bildnisse, zum mitfühlenden und aufklärenden Nachvollzug des Lesers, zu seiner Selbstüberprüfung und seiner geschichtlichpolitischen Selbstvergewisserung wie geschaffen.

\section{Struktur der Erinnerung}

Das ästhetische Medium des Ich-Erzählers ist die Erinnerung. Wie in den letzten Werken Uwe Timms, Rot und Am Beispiel meines Bruders, gewinnt sie auch hier eine faszinierende Qualität. Der literarische Akt des Sich-Erinnerns wirkt lebensnah, er ahmt das Leben kunstvoll-unaufdringlich nach. " Hin und wieder ", so bemerkt der IchErzähler eingedenk des Freundes, « begegnet er mir ganz unverhofft, und jedesmal ist er mir dann nah, in seinem Lachen, seinem Nachdenken ». Analog zum « unverhofft », zur mémoire involontaire, bietet der Ich-Erzähler seine Erinnerungsarbeit dar (S.) : also achronologisch und assoziativ-sprunghaft, wie die Erinnerung sich im wirklichen Leben vollzieht. Die Assoziationen und zeitlichen Sprünge erfolgen so, daß der Eindruck von Lebensdichte entsteht. Die Zeiträume der Erinnerung wirken dank des Verzichts auf eine chronologische Reihung gleich nah, gleich gegenwärtig. Erste Schuljahre und Lehre des Ich-Erzählers, zweite Schulzeit und Freundschaft mit Benno Ohnesorg, Studienaufenthalt in Paris und Konfrontation mit dem Tod des Freunds, die Protestbewegung danach, die späteren Erkundungen über sein Vor- und sein Nachleben: alle Lebensabschnitte erhalten durch die hin und her schwingende Erinnerung dieselbe Nähe, dieselbe Präsenz. Die Sprünge von einer Zeitebene in die andere, etwa von einer entfernten Vergangenheit in die vorweggenommene Zukunft sie enthalten Assoziationskräfte, die des Lesers Phantasie zur Verbindung des chronologisch Unverbundenen zwanglos anregen. Zutage tritt Wesentliches: die Gleichzeitigkeit des Ungleichzeitigen. In der erzählten Dynamik sich übereinander 
lagernder und sich durchdringender Zeiträume erscheint das Leben als die unabgeschlossene komplexe Einheit von Lebensphasen, die einander ablösen und gleichwohl miteinander verknüpft bleiben.

\section{Woanders - anderswo}

5 Im epischen Prozeß der erinnerten und sich übereinander lagernden Lebensphasen tritt eine allmählich in den Hintergrund - die primäre Schul- und Lehrlingszeit des IchErzählers. Obgleich der zunächst eingeschlagene Weg zur Ausbildung als Kürschner ihm fürs erste eine materielle Lebenssicherung bietet, obgleich die handwerkliche Arbeit des Kürschners eine gewisse Verwandtschaft mit dem Handwerk des schreibenden Stilisten aufweist (vgl. S. 30f.), ist dieser Lehrling, der " jeden Auftrag vergaß, der durch den Tag stolperte oder wie narkotisiert herumstand » (S. 29), ganz "woanders ", so sein eigenes Wort (S. 25). «Woanders", also das Sinnen und Trachten auf ein "Anderswo" gerichtet, einen "absonderlichen Weg", den die KürschnerGesellen, als er ihn einmal verrät, mit "Gelächter» abtaten (S. 29). Es ist der Geheimweg zum Schriftsteller. Der Lehrling hängt «Tagträumen» nach (S. 26) und sucht «Worte, Worte, die das ausdrücken sollten, was noch eine sprachlose Empfindung war » (S. 27) ; nur ein Kürschner-Meister, literarisch interessiert, ermutigt ihn, ein "Meister der Freundschaft» auch, der dem jugendlichen Außenseiter mit feinem Gespür zur Seite steht (S. 29).

Das ersehnte Anderswo nimmt eines Tages Form an, eine Vorform zumindest. Wie ein entscheidender Schritt zum Schriftsteller-Beruf erscheint dem Suchenden das Braunschweiger Kolleg für Begabtenförderung, das Nichtabiturienten mit abgeschlossener Lehre als Stipendiaten zum Abitur führte und ihnen damit ein Studium ermöglichte. "Ich war davon überzeugt », so der Ich-Erzähler im Rückblick, «daß ich für das Schreiben, so wie ich mein Schreiben verstand, eine akademische Ausbildung brauchte, philologische, philosophische Kenntnisse» (S.49). Es ist ein Aufbruch zu neuen Ufern, erlebt mit einer Intensität, die (nach meiner eigenen Erfahrung mit Spätabiturienten) nur Menschen entwickeln, die bislang beruflich eingespannt, Tag für Tag gefordert waren. Ihnen winkt eine ungekannte Freiheit :

Einen Überfluß an Möglichkeiten, das war es, was uns das Kolleg nach mehreren Jahren Arbeit in einem ungeliebten Beruf bot, plötzlich erschien alles offen, ein Gefühl der Erweiterung, der Erleichterung, der Genuß, dieser Selbstgenuß, sich mit dem Eigenen beschäftigen zu können. Angeregt durch Lehrer - wenn sie denn selbst von ihrem Stoff angeregt waren -, war das freiwillige Lernen fast immer eine Lust, die Lust, sich neu $\mathrm{zu}$ finden. Ein Lernen auch aus dem täglichen Zusammenleben mit anderen [...] Sie hatten in ihren Berufen gearbeitet, und etwas in ihnen hatte brachgelegen, der Wunsch nach sozialem Aufstieg trieb sie, sicherlich, aber auch noch etwas anderes, jedenfalls bei einigen, was sich mit dem alten Wort Berufung bezeichnen läßt. Sie wollten genau das studieren, was sie seit langem, oft schon seit der Kindheit interessierte, und die meisten wurden, was zu werden sie sich wünschten: Wissenschaftler, Ingenieure, Ärzte, Theologen, Biologen, Psychologen, Lehrer, Manager. (S. 56f.)

7 Die beschwingte Prosa gemahnt an die lyrische Verve eines Friedrich Nietzsche; sein Gedicht Nach neuen Meeren setzt mit den Versen ein :

Dorthin - will ich ; und ich traue

mir fortan und meinem Griff. 
Offen liegt das Meer, ins Blaue

treibt mein Genueser Schiff. verlockende Fernziel nur über Umwege näherrückt. Und es nur aufgrund einer zufälligen Information - «einer der das Leben bestimmenden Zufälle» (S. 49) überhaupt in den Gesichtskreis treten läßt. Nicht auf eigenem Verdienst allein, auch auf der Gunst des Zufalls kann das Gelingen beruhen. Und vielleicht auch um Haaresbreite verfehlt werden. Noch bevor das Kolleg an seinem Lebenshorizont aufgetaucht war, hatte den Ich-Erzähler « das Gefühl » beunruhigt, « abzudriften von dem, was ich für mich als bestimmend hielt» (S. 49). Damals hatte er seine freie Zeit mit einem fidelen Kumpel verbracht, der ihn als angehenden Kunstwissenschaftler in die feinere Gesellschaft einführte, worauf der Kürschner-Geselle sich flugs, in der Manier eines Tausendsassas, die seriöse Miene und Rhetorik eines Kenners in aestheticis aneignete und damit erfolgreich bluffte - wie weiland Felix Krull. Die Episode stellt sich im Rückblick als eine charmante Abweichung vom Weg in die Selbstbestimmung dar : «Ich weiß seitdem, daß ich auch eine Karriere als Hochstapler hätte machen können. » (S. 48).

Er fängt sich rechtzeitig; "ein zartes, aber bestimmtes Gewissen meldete sich ", erweckt durch die «Erinnerung an die eigenen Tagträume » (S. 47). So fein ist das Gespinst, das ihn zu seiner Berufung zurückführt, über den Weg ins Kolleg. Dort trifft er auf einen Klassenkameraden, der sich für Literatur interessiert und selbst Literarisches zu Papier bringt: Benno Ohnesorg. «So begann es, daß wir einander unser Geschriebenes zeigten und er mein erster Leser wurde. » (S. 9)

Lesung des Selbstverfaßten, Lektüre klassischer und moderner Werke, Verständigung über aktuelle Probleme literarischer Sprache, Erkundungen des Rhythmus und der Melodie in «Wortfolgen» (S. 37), Herausgabe einer Zeitschrift - solche gemeinsamen Unternehmungen bringen die beiden Kollegiaten einander nahe und stiften ihre Freundschaft, wobei Temperament und Charakter des Freunds als produktiver Antrieb wirksam sind und Vertrauen erwecken, ohne besitzergreifend zu sein. Der ethische Anspruch, den Ohnesorg an sich selbst stellt, « frei von Eigenliebe und Geltungsdrang » zu leben (vgl.S. 19), entspricht offenbar einer Wesensart, deren «sanfte Zurückhaltung sich jeder offen ausgetragenen Konkurrenz verweigert[e]» (S. 79) und im Dialog nicht den Anflug einer « Verstimmung » aufkommen läßt (S. 168). So wird jener emphatische Augenblick möglich, der eines Tages zur dialogischen Selbstdarstellung und Selbstverpflichtung führt :

Einmal, im Sommer des zweiten Jahres, als wir an der Oker saßen, in der Stille eines sich langsam auftürmenden, durch keinen Windstoß sich ankündigenden Gewitters, und uns über Gefühl und Sprache unterhielten, über uns, in dem eben noch blauen Himmel, grauschwarz das tiefhängende Gewölk, las er mir, was er sonst nie tat, ein nicht fertiges Gedicht vor. Noch fehlte die letzte Strophe. Einer Ode gleich war der Klang, der Rhythmus, den ich noch immer höre, diesen Gesang, ohne mich an eine Zeile, auch nur an ein Wort erinnern zu können. Als er geendigt hatte, sagte keiner von uns ein Wort, und wir sahen den Regen näher kommen, eine dichtfallende gesträhnte graublaue Front, in der die Blitze niederfuhren, und der Donner rollte über die Wiesen und Felder heran, mit einer Wucht, die sich auf die Brust legte, einen Moment gelähmt, nicht vor Schreck, sondern innig beglückt standen wir und wurden in diese Flut eingetaucht.

Es war der Augenblick, in dem wir wußten, es wird gelingen, das Selbstgewählte voranzubringen, mit allem und jedem Einsatz, allein dem verpflichtet. Kein Unbeteiligtsein, sondern darin aufgehen. Ein hoher Ton, der nur an dem Beginnen 
möglich ist und sich nur aus der Unschuld der verspätet sich Bildenden erklärt, aus Traum und Wunsch, die man sonst niemandem hätte anvertrauen können. Schreiben nicht als Alternative, nicht, weil man es gern möchte, aber doch auch etwas anderes tun könnte, sondern weil man keine Wahl hat, dieses Eingeständnis, das von ihm nicht nur mit Verstehen beantwortet wurde, sondern mit brüderlichem Gleichsinn und Vertrauen. Eingestanden auch, daß dieses Müssen eine Selbstverpflichtung war, eine Gegenwehr, deutlich schon als Kind spürbar, schreiben $\mathrm{zu}$ müssen, was aus schulischer Qual erwuchs und doch von geheimnisvoller, ahnungsvoller Lust begleitet war. (S. 70f.)

11 Wer sich der Tradition literarischer Berufung und Selbstverpflichtung erinnert, der wird die dargestellte Szenerie - das Bekenntnis des poetischen Auftrags im dramatischen Naturraum - mit älteren Texten assoziieren - etwa Hölderlins Wie wenn am Feiertage... Mit dem Unterschied, daß sich hier zwei auf den Weg zu ihrem Fernziel machen - jenem Anderswo, das ihr zentrales Lebensmotiv ist. So gelangen sie über die gleichartige handwerkliche Ästhetik ihrer Lehrlingsjahre - der eine betrieb sie als Kürschner, der andere als Dekorateur (vgl. S. 15) - gemeinsam hinaus.

Für den Realitätssinn des Ich-Erzählers spricht, daß er das jahrelange Verstummen der Freundschaft nach der Kollegzeit nicht überspielt, für seine subtile Psychologie, wie er es deutet. Sechs Jahre lang gehen die Freunde getrennte Wege, ehe der eine, der spätere Ich-Erzähler, vom anderen wieder hört: in Form der Todesnachricht. Ein herber Schlag. Waren die getrennten Wege die Ursache für ihr Verstummen auch als Schriftsteller? Fehlte die inspirative Nähe des jeweiligen Gegenübers? Niemand von den Berliner Freunden Benno Ohnesorgs kann ihm poetische Produktivität nachrühmen, auch seiner Frau ist nichts aus seiner Feder bekannt. Und der IchErzähler selbst? Er weiß nur von dem «Stillstand» allen Schreibens in München zu berichten, wohin es ihn nach dem Kolleg-Abitur zog (S. 75) : Stillstand aufgrund der «Gewohnheit, der Festschreibung, dem Eingeschliffenen, der Ermüdung im Studium, der Gleichgültigkeit gegenüber Künftigem. [...] es hatte sich etwas von dem Antrieb zum Schreiben verloren, [...] zum Schreiben von Gedichten und Prosa " (ebd.). Das Grundmotiv ihrer Existenz war offenbar beiden aus dem Sinn und der Hand geglitten, das ersehnte Fernziel, das Anderswo, hatte sich verflüchtigt. Die Schuld dafür schreibt sich der Ich-Erzähler zu; die spätere Aussage von Ohnesorgs Frau, ihr Mann habe mit ihm, dem fortgezogenen Freund, " nach unserem Abschied gehadert » (S. 13), mag ihn nun, beim Schreiben, zu einer intensiven Selbstbefragung, ja Gewissenserforschung veranlassen :

Erst langsam und während dieser Erinnerungsarbeit ist mir deutlich geworden, wie sehr ihn getroffen haben muß, daß ich unseren ursprünglichen Plan, gemeinsam nach Berlin zu gehen, kurzfristig änderte und zum Studium nach München zog. Die Lust des neuen Anfangs. Spontan war die Entscheidung gefallen und mit dem Freund nicht abgesprochen. Es muß ihm als Verrat an unserer Freundschaft erschienen sein, was mir eine romantische Vorstellung war: die Trennung nach den langen Braunschweiger Gesprächen, um irgendwann einmal literarisch voneinander zu hören, voneinander $\mathrm{zu}$ lesen, und sich dann - erst dann wiederzusehen. Eine Trennung als literarische Bewährungsprobe. (S. 113)

So wäre denn der Ich-Erzähler, der in die Fremde zog, zum « Fremden » geworden ? Die Erzählung, die als « Fremden » den Freund zu bezeichnen scheint, läßt doch den IchErzähler nicht aus dem Spiel mit dem anonymen Namen. Dafür spricht auch die subtile Psychologie seines Bekenntnisses: Es verrät ein strenges Gewissen, das über die Trennung im Nachhinein befremdet ist. Und doch: War für die Trennung nicht auch ein zeitspezifisches Geistesklima mitverantwortlich? Zum Beispiel die verbreitete 
«Vorstellung vom Unabhängigsein » (S. 79) und von der « indifférence » (S. 97), wofür damals namentlich die französische Literatur und Philosophie Modell stand ? Es ist der Ort, darauf einen Blick zu werfen.

\section{Wegkreuzung. Deutsch-französische Interkulturalität}

Vor seiner Kollegzeit hatte der Freund Frankreich bereist, französische Literatur, Kultur und die Provence kennengelernt; im Kolleg verfaßt er einen Aufsatz über die fragmentarische Form bei modernen französischen Schriftstellern (vgl. S. 153). Wie den Ich-Erzähler bewegt ihn damals das von Jean-Paul Sartre aufgeworfene Problem der Freiheit, das für beide, die aus ihrem erlernten Beruf heraustreten, um neue Wege zu beschreiten, weg-weisend ist: jene Freiheit, die einem die Wahl zusprach, «einen anderen aus dem zu machen, der aus einem gemacht worden war und den man aus sich selbst gemacht hatte» (S. 56). Während der zwei Jahre im Kolleg tauschen sich die beiden Freunde wiederholt über literarische Werke und Kulturphänomene des Nachbarlandes aus, lesen gemeinsam L'Étranger (Der Fremde) von Albert Camus und befassen sich mit seiner Konzeption des Sisyphos-Mythos (vgl. S. 95).

Timms Erzählung bringt damit einen aufschlußreichen kulturgeschichtlichen Sachverhalt ins Spiel : die Entdeckung Frankreichs durch die Jugend-Generationen der Nachkriegsepoche, jenes Frankreich, das der « Vätergeneration » noch als Land der « Erbfeinde " gegolten hatte (vgl. S. 72) :

Das wirkliche Frankreich zu erleben, sich diesem Land, seiner Kultur zuzuwenden, dazu entschlossen sich viele meiner Generation, sie brachen auf als Aupair, als Austauschschüler oder als Hilfskräfte bei der Wein- oder Obsternte und belebten so den Freundschaftsvertrag zwischen den beiden Ländern. (S. 72f.)

Sozialpsychologisch ist daran bedeutsam, daß deutsche Jugendliche im Medium der Fremde sich von den Befangenheiten und Konventionen der Eltern-Generation lösen und ihrem eigenen Lebensstil eine andere, freiere Note verleihen wollten. Wer - wie Uwe Timm - zwischen 1935 und 1945 geboren wurde, wird sich nicht ohne peinliches Gefühl an gewisse Verhaltensweisen der Eltern-Generation erinnern - an ihre Prüderie, ihren Konformismus, ihre provinzielle Enge, ihre auf Wohlverhalten erpichten Erziehungsmaximen, ihre Neigung zu Plüsch und Biederkeit, ihre Förmlichkeit, ihre Fremdenscheu, die nicht der geringste politische Luftzug durchwehte, ihre betuliche, an Sentimentalitäten und Heimatfilmen sich erwärmende Gefühlskultur, allerdings auch an ihren Arbeitseifer, der einem lange entbehrten Wohlstand zugewandt war, und nicht zuletzt auch an ihre autoritären Gepflogenheiten. Daß manche dieser Züge einer Lebenslüge entspringen konnten, einer geflissentlich verschwiegenen politischen Vergangenheit, merkten wir, die apolitisch erzogene junge Generation, erst spät am Ausgang der sechziger Jahre. Aber auch ohne diese Einsicht tat eine Befreiung von der als unleidlich empfundenen Konventionalität älterer Erwachsener not. Wer damals das Glück hatte, mit literarischen Aktualitäten in Frankreich bekannt zu werden, etwa dem Frühwerk von Albert Camus, konnte sich in eine "ästhetisch-moralische Revolte " (S. 91) zumindest hineinphantasieren. Just dies tun die beiden Freunde im Braunschweig-Kolleg, und wohl kein Werk konnte sie suggestiver dazu überreden als L'Étranger von Albert Camus :

Ich hatte das Buch, als ich an das Kolleg kam, eben zum zweiten Mal gelesen und las es mit ihm gemeinsam zum dritten Mal. [...] Dieser stereoskopische Blick auf Dinge und Menschen. Die Genauigkeit in der Beschreibung der Gefühle. Keine Heuchelei, 
keine Selbsttäuschung, keine Kompromisse, keine verschwiemelte Sinngebung, das gefiel uns, ihm, mir und vielen anderen an den Büchern von Camus. Eine Lektüre, die in dem Alter der Selbstfindung ihre Kraft entfaltet.

Was uns in Der Fremde ansprach, war die Abgrenzung von all dem, was Konvention war, die Infragestellung der großen Gefühle und Tugenden: Nation, Familie, Heimat, Pflicht, Glaube, Treue. Das hatten wir herausgelesen, die Kühle, den Zweifel, keine Gewißheit, den Wunsch nach Konsequenz, die Leidenschaft Denken, das vor allem, sich nicht vorschnell mit Widersprüchlichem zu versöhnen, keine Lauheit dulden. Bindungslosigkeit und Gleichmut waren dafür die Voraussetzung. Die indifférence war der geheime Treibsatz [...]. (S. 64f.)

Gleichmut also im Bunde mit Unabhängigkeit ist die Botschaft, die man dem Roman von Camus abgewinnen konnte: Bereitschaft daher auch zur Trennung von nahestehenden Menschen aus dem Gefühl einer sich selbst genügenden, bindungsfreien Individualität. Viel spricht für die Annahme, daß diese zunächst literarisch-philosophische Neigung ins Leben selbst einzog und eine Zeit lang die Mentalität mitbestimmte. So wird denn auch die tapfere und von Schmerz nicht freie Trennung verständlich, die der Ich-Erzähler zum Ende seiner Kollegzeit sich selbst und dem Freund zumutet. Die gemeinsame intensive Bindung an das literarische Werk wird zum Grund ihrer Ent-Bindung und jahrelangen Entfernung : ein eigentümliches Paradox. Habent sua fata libri...

Die Geschichtsauffassung des frühen Camus ist unpolitisch. Denn die Geschichte, die als sinnlos begriffen wird, ist politischen Interessen und politischem Veränderungswillen unzugänglich. Sie verweigert sich der um eine Sinngebung bemühten Vernunft und spottet einem auf Strukturen und Zusammenhänge bedachten Erkenntniswillen. Darin sieht Camus das Absurde des Daseins. "Das Absurde entsteht aus diesem Zusammenstoß zwischen dem Ruf des Menschen und dem vernunftlosen Schweigen der Welt. " (Camus, zit. nach der deutschen Übersetzung, S. 95). Die späteren Werke des französischen Schriftstellers, La Peste und L'Homme révolté, weisen über die indifférence des Menschen hinaus. Angesichts des Leids in der Welt sieht sich das Individuum zum Handeln und solidarischen Eingreifen veranlaßt. Dem individuellen Gleichmut folgt das soziale Engagement des Einzelnen: aus der «ästhetisch-moralischen» wird die gesellschaftsbezogene «Revolte». Der « Mensch in der Revolte», so pointiert es der Ich-Erzähler, « anerkennt als einzig verbindlichen und verbindenden Wert den Kampf gegen den Tod, gegen Qual und Unterdrückung, im Namen einer diesseitigen freien Brüderlichkeit. Es gibt nur dieses eine Leben, im Hier und Jetzt, darum die rebellische Beschwörung des Lebens, in deren Mittelpunkt die Freiheit und die Verantwortung des einzelnen stehen. » (S. 92)

Diese Einsicht gewinnt der Ich-Erzähler erst im Laufe der Jahre, und aktive Impulse entwickelt sie erst während seines Studienaufenthalts in Paris 1966/67 und vor allem bei seiner Rückkehr nach München im Gefolge der Protestbewegung. Davor, in den fünf Jahren, die auf seinen Abschied von Braunschweig folgten, war er an die unpolitische Perspektivlosigkeit gebunden, die dem Frühwerk von Camus anhaftet. Die Mutmaßung drängt sich auf, daß dies einer der Gründe für sein Verstummen als Schriftsteller war, neben den schon angeführten Motiven: der Trennung vom Freund und der Verlagerung seiner Geisteskräfte auf das Studium. Ein allgemeines Zeitklima, das dem Handeln von vornherein mit Skepsis begegnet und ihm einen geschichtlich konstruktiven Sinn abspricht, kann die Produktivkräfte eines noch nicht anerkannten, noch im Werden begriffenen Schriftstellers behindern. Seine Berufung, die Suche nach dem Anderswo, das über die bestehenden Zustände hinausführt, droht zu versiegen. 

unter Generalverdacht. Es läßt sich nicht einfach aus der Welt schaffen, auch dann nicht, wenn die besten, sinnvollsten Absichten verfolgt werden. Der unberechenbare Geschichtsverlauf kann sie von Fall zu Fall ad absurdum führen. Die Tötung Benno Ohnesorgs bezeugt dies. "Im gemeinsamen Protest, in der Revolte, wurde die indifférence, meine, vieler, überwunden. » (S. 93) So bemerkt der Erzähler zu Recht. Und so fährt er fort : «Wie Camus durch seine Mitarbeit in der Résistance seinen Sinn fand, so fand sich Sinn in dem Protest gegen den Staat » (ebd.). Wo aber ist der Sinn im Tod Ohnesorgs zu finden? Nirgendwo.

Das führt uns zum vorletzten Abschnitt.

\section{Tatort (2) Kein Ort. Nirgends ?}

«Protest gegen den Staat »- damit ist ein Ferment jenes Zeitklimas bezeichnet, das beim Tod Benno Ohnesorgs in der jungen Generation vorherrschte. Welche Motive aber lagen der Politisierung ihrer «ästhetisch-moralischen Revolte » zugrunde? Der IchErzähler erinnert an die Entstehung ihres geschichtlichen Bewußtseins, eines die «Väter » infrage stellenden Bewußtseins, jene Väter,

die gehorsam ausgezogen waren, die Welt zu erobern und den Krieg verloren hatten, die wissentlich oder nicht wissen wollend am Völkermord an Juden, Sintis und Roma beteiligt waren, auch dann, wenn sie nur tapfer gekämpft oder fleißig in der Rüstung gearbeitet hatten, die sich nach dem Krieg in die Umerziehung hatten schicken müssen, widerwillig, ihre befehlsgewohnte Lebensweise jedoch zäh in den Familien, Vereinen, Parteien behaupteten, Gehorsam forderten, die Lehrer, Richter, Staatsanwälte, Offiziere, die alle noch gedient hatten, Parteimitglieder gewesen waren und sich nun unter dem Druck der Siegermächte im Westen demokratisch wendeten. [...] Gegen diese Elite, gegen das Establishment, das von meiner Generation wie eine Besatzungsmacht empfunden wurde, richtete sich die Revolte. (S. 90f.)

Es war eine bis $\mathrm{zu}$ Ohnesorgs Tod zwar verbreitete, im studentischen Milieu anwachsende Revolte gewesen, aber auch wenig zielstrebig, mit einzelnen, voneinander isolierten Brennpunkten, eine Protestbewegung, die bei besonderen Anlässen - wie etwa dem Schah-Besuch - aufflackerte und dann, von der Staatsmacht gezügelt, weiterschwelte. Ohnesorgs Tod jedoch, darauf beharrt der Ich-Erzähler entschieden,

verwandelte den schon vorhandenen, aufgestauten Unwillen in den Willen zur Tat. Die Zeit war, wie es heißt, reif. Aber damit es zu solchen Wetterschlägen kommt, ist eine besondere Situation nötig, eine besondere Person, ein besonderes Bild, das sich im Bewußtsein verankert, das Erkennen mit dem Gefühl auflädt, was wiederum die analytisch gewonnene Erkenntnis befeuert. (S. 118)

Wäre das der historische Sinn des « Opfertodes » Ohnesorgs gewesen? Mit dieser Frage bewegt sich das Erkennen auf einen Abgrund zu. Ist es denkbar, daß ein Menschenleben den Status eines sinnvollen Opfers gewinnt, indem sein katastrophales Ende zum Fanal der Kräftigung, Bündelung, Radikalisierung einer politischen Bewegung wird, zum Fanal auch der Erweckung vieler unentschiedener oder passiver Einzelner? Der Ich-Erzähler spitzt die Frage dramatisch zu, er verleiht ihr einen herausfordernden Akzent, wenn er die gewaltige, medial potenzierte Wirkung dieses 
Todes wiederbelebt und sie mit einer revolutionären These des einflußreichsten Ideengebers damals, Karl Marx, verknüpft :

Er hat viel bewegt - als Opfer. Das Foto, das ihn am Boden liegend zeigt, das in allen Zeitungen zu sehen war, das immer wieder abgebildet wurde, das ich in Paris sah, [...] dieses Foto hat, wie nur Bilder es vermögen, Empörung erzeugt. [...]

Bilder, die sich ins Bewußtsein einsenken, eine hochverdichtete, aus sich heraus sprechende Situation zeigen und so rationale Einsichten emotional aufladen und an die eigene Handlungsfähigkeit appellieren. Ein Kraftstoß, der nicht durch Einsicht immer wieder erneuert werden muß, sondern langanhaltend wirkt : Du mußt dich ändern. Du mußt etwas tun. Es genügt nicht, daß der Gedanke zur Verwirklichung drängt, die Wirklichkeit muß sich selbst zum Gedanken drängen. Ein Satz aus den Frühschriften von Karl Marx. (S. 117)

So viel zum symbol- und sinnträchtigen Tatort, an dem Ohnesorgs Leben zum Erliegen kam.

Dennoch - welche politisch-gesellschaftlichen Energien auch immer durch die Initialzündung seines Tods ausgelöst wurden, welche Assoziationen mit einer revolutionären Idee diese Zündung auch immer heraufbeschwor : rechtfertigen läßt sich damit Ohnesorgs Tod nicht. Ein einziger schlichter Satz hebt alle historische Sinngebung auf: "Nicht sein Leben und Schreiben sollten ihn bekannt machen, sondern sein Tod.» (S. 113) Das Leben Ohnesorgs war auf anderes ausgerichtet als auf ein frühes, gegen seinen Willen herbeigeführtes Ende - vielleicht auf das Schreiben nach wie vor, vielleicht auf die Familie mit seinem noch ungeborenen Kind, vielleicht auf Kunsterziehung, und sehr wahrscheinlich auf jene Ethik, die er schon früh als seine Lebensmaxime formuliert hat: daß, mit Brecht $\mathrm{zu}$ sprechen, «der Mensch dem Menschen ein Helfer » sei. "Absurd" ist der Geschichtsverlauf, wenn er einen Menschen willkürlich, gegen dessen Willen, zerstört, einen Gipfel der Absurdität erreicht er, wenn er ausgerechnet einen Helfer der Menschen aus dem Weg räumt, mit brutaler Gewalt den vernichtet, der keines Menschen «Feind» gewesen war, den jeder, der ihm begegnete, « mochte » (S. 84).

Das «Empörende an seinem Tod", so bekräftigt der Erzähler, sei « das Absurde ». (S.113) Es entsteht indes eine weitere, eigentümliche Paradoxie, wenn er feststellen kann, daß die - von Ohnesorgs Schicksal mit ausgelöste - «Wendung » des Protests « ins Politisch-Aufklärerische » einen " gesellschaftlichen Sinn » konstituierte, an dem er, der Student und Doktorand in München, von jetzt an teilhaben kann. Das "Absurde », worüber er sich gemeinsam mit dem Freund, lesend und diskutierend, verständigt hatte in der Kollegzeit, löst sich nun in der solidarisch-politischen «Revolte» auf (S.130). Der davon ausgesperrte Freund blieb hingegen an die Absurdität des Geschichtsverlaufs durch seinen Tod gefesselt.

Nicht nur in dieser Hinsicht ist der Freund auch der "Fremde", der im Titel der Erzählung genannt wird. Er ist dies auch in der merkwürdigen Beziehung, die er zu Camus' L'Étranger unterhält. Mit seiner Neigung zur Introversion (zitiert nach Timm, S. 123) " hielt er sich», wie Meursault, Camus' Protagonist, "vom geselligen Treiben fern ». (S. 39) Nonkonformist, der er wie selbstverständlich ist, teilt er Meursaults reservatio mentalis gegenüber Konventionen. Freilich ist seine Nähe zu dem Fremden im französischen Roman auch eigenartig gebrochen. Die Deutung, die er den Todesschüssen Meursaults auf den Araber in seiner Nähe gibt, ist der «Urteilsbegründung» ähnlich, die «zum Freispruch» des Zivilpolizisten Kurras, Ohnesorgs «Todesschütze », führen sollte : «Es hat sich sogar nicht ausschließen lassen, 
daß es sich bei dem Abdrücken der Pistole um ein ungesteuertes, nicht vom Willen des Angeklagten beherrschtes Fehlverhalten gehandelt hat. » (S. 92) Hätte folglich Ohnesorgs literarische Deutung seinen eigenen Todesschützen salvieren können? Der IchErzähler scheint die Herstellung von überraschenden Korrespondenzen zu lieben. Wahr aber bleibt in diesem Zusammenhang, daß in Camus' Roman das Gericht den Protagonisten verurteilt, während die deutsche Justiz den Freispruch des Zivilpolizisten offenbar aus politischen Motiven, der «staatlichen Gewalt » zuliebe, herbeiführte : ein Grund mehr für die Radikalisierung der Protestbewegung (S. 92f.).

An überraschenden Korrespondenzen, Querverweisen, Spiegelungen und Gegenspiegelungen ist die Erzählung reich genug - ein Zeugnis für Uwe Timms artistisches Bewußtsein. Benno Ohnesorg ist «vor dem Todesschuß zusammengeknüppelt worden", " elf Blutunterlaufungen verzeichnet der Obduktionsbericht » (S. 166 u. 167). Der Leser horcht auf, nachdem der Erzähler zuvor Hinweise eingestreut hat auf den späten Nietzsche, für den sich Ohnesorg zu interessieren begann, weil der «in Turin einem vom Kutscher geprügelten Droschkengaul um den Hals gefallen [sei] und Ecce Homo gerufen» habe (S. 150). Ferner erzählt Ohnesorg einmal von schwer beladenen darniederliegenden Kamelen, die sich « unter den immer heftigeren Schlägen » ihrer Treiber « mit einem ächzenden Stöhnen, in dem alles Leid der Welt versammelt schien ", mühsam erhoben hätten (S. 150f.). Alles Leid der Welt - erfaßt es später auch den zusammengeknüppelten Ohnesorg? Wenn er sich mit dem Anarchisten Gustav Landauer befaßt, « der nach dem Ende der Münchner Räterepublik im Gefängnis von Freikorpsleuten zu Tode geprügelt worden war » (S. 154) : nimmt er dann seismographisch, in brüderlicher Empathie, sein eigenes Schicksal vorweg? Ist es das ihm eigentümliche, als "Fremden" in der menschlichen Gesellschaft ihn auszeichnende «meditative Versunkensein » (S. 41), das es ihm ermöglicht, das Leid der Welt so intensiv zu spüren, wie es die Protagonisten in Camus' La Peste erfahren, so daß er sein Lebensende am eigenen Leib, kraft der ihm eigenen Sensibilität, vorwegempfindet?

Der Ich-Erzähler gibt dem Leser beziehungsvolle Rätsel auf, die ihn während und nach der Lektüre nicht ruhen lassen. Er zeichnet in die Gestalt des «Insichgekehrten » (S. 8) einen aggressiven «Ausbruch » ein, ein tobendes «berserkerhaftes Zwiegespräch mit einem Niemand» (S. 86). Er läßt den jungen Mann, der so sehr auf die eigene Freiheit und die Unantastbarkeit der Mitmenschen bedacht ist, als Betrunkenen in einen ungehemmten Tanz mit der Freundin eines Kollegiaten geraten und beide « in einem ekstatischen Wirbel zu Boden » stürzen (S. 96). Warum? Überforderte Ohnesorg die eigene vorbildliche Selbstkontrolle, die der "Prozeß der Zivilisation" von den Menschen verlangt? War er deshalb einmal, zweimal jenen Affekten ausgesetzt, deren Dämpfung gerade er sonst beispielhaft vorlebte? Konnte er deshalb als Leser, der die Gefahr auszubrechen kennt, die Aufwallung der Impulse und Emotionen Meursaults nachvollziehen, die ihn zu seinen Todesschüssen auf den Araber verführt?

Die Rätsel und Ungewißheiten, die den lebenden Benno Ohnesorg umspielen, reichen über den toten hinaus. Der Berliner Hinterhof, auf dem die Fotos ihn tausendfach zeigten, stellte einen Unbehausten aus. Vom Grab, in dem er ruhen sollte, wurde der Grabstein entfernt und nicht wieder zurückgebracht: vielleicht weil die Ehefrau und der Sohn dem Namen, Benno Ohnesorgs Namen, der sie an seinen Verlust und seine langwährende Wirkungsgeschichte erinnerte, zu « entkommen » suchten (S. 136f.). Der Sohn konnte dem Toten keine Ruhestätte, konnte ihm nur einen Ersatz dafür 
gewähren: eine Kammer voller unbrauchbarer Gegenstände, die er sammelt «als Ersatz für das, was er verloren, nie kennengelernt hat, den Vater, aber auch für das, was die Mutter verloren hat " (S. 133). Das Porträt, das der Ich-Erzähler von Lukas Ohnesorg, seiner Kammer und seinem verlorenen Vater entwirft, ist eines der zwingendsten in dieser mit faszinierenden Porträts ausgestatteten Erzählung. So erscheint denn der tote Benno Ohnesorg als Obdachloser. Kein Ort. Nirgends?

Findet der werdende Schriftsteller, dem die « Wortlandschaften » (S. 38) nicht zum Ort der ersehnten Identität wurden, auch fernerhin keine Ruhe? Die Gegenfrage führt uns weiter : Was wurde im Lebensgang des Ich-Erzählers aus jener literarischen Berufung, die ich hier als das Anderswo bezeichnet habe und die beide Freunde im intensiven Dialog erprobt hatten? Wurde sie Realität nach dem jahrelangen Verstummen, das auf die Trennung vom Freund folgte? Es gibt auf die Frage eine indirekte Antwort: die vorliegende "Erzählung ». Sie ist die Gedenkschrift, die den Freund noch einmal zum Sprechen bringt, ist die Dankesschuld, die der Ich-Erzähler ihm gegenüber abträgt, dem Verstummten, Sprachlosen, an den kein Grabstein mehr gemahnt. Vielleicht findet er eine « bleibende Stätte » (Hölderlin, Mein Eigentum) in dem Buch, das so beredt von ihm erzählt und ihm, dem Zeitgenossen der sechziger Jahre, eine unverjährte Gegenwart bezeugt. Der « reife Gesang ", der ihm versagt wurde, blieb dem Freund, dem IchErzähler, nicht vorenthalten; dem Toten zu Ehren hat er ihn verfaßt, aus der Erinnerung an ihn ist er erwachsen. Der Erinnerte hat durch seine intensive Präsenz daran mitgeschrieben. So könnte von dem ruhelosen "Orkus ", den wir eingangs aus Hölderlins An die Parzen zitiert haben, Ohnesorg zuletzt doch in die "willkommene" «Stille der Schattenwelt » gelangen.

\title{
Der dritte, deutsch-französische Weg
}

\author{
Sagen Sie \\ Ihm, daß er für die Träume seiner Jugend \\ Soll Achtung tragen, wenn er Mann sein wird. \\ (Schiller : Don Carlos - Marquis Posa zu Königin Elisabeth als sein Vermächtnis für \\ Don Carlos)
}

Die Erzählung verrät die Träume einer Jugend nicht, die inzwischen im reifen Alter angekommen ist - weder den heroischen Traum vom Absurden (Camus) noch den einer befreiten Gesellschaft (Marx). Daß letztere ein Ziel geschichtlichen Handelns bleibt, daß nach den Worten von Marx « alle Verhältnisse umzuwerfen (sind), in denen der Mensch ein erniedrigtes, ein geknechtetes, ein verlassenes, ein verächtliches Wesen ist ", hebt der Erzähler hervor (S. 159), indem er im selben Atemzug den Anspruch formuliert, sich selbst zu verändern, wenn man die «Verhältnisse zu ändern » wünscht (ebd.), ein namentlich von Herbert Marcuse vertretener, in den späten sechziger und frühen siebziger Jahren vieldiskutierter Anspruch. Von hier aus führt ein Weg zu Camus' späteren Werken, vor allem zu L'Homme révolté, jener vom Ich-Erzähler in der « zweiten Fassung » seiner Dissertation über Camus beschrittene Weg (ebd.). Er verfolgt ein « auf Veränderung zielendes Philosophieren », das "auf den Anspruch eines Glücks im Diesseits für alle insistiert » (ebd.). Gemeint ist ein persönliches Glück, das auch « das Glück der anderen» einschließt: «darum der Anspruch auf Freiheit und Gerechtigkeit », auf "eine distinktive Wahrnehmung von Herrschaftsmechanismen, von Unrecht, von eklatanter und stiller Ausübung von Gewalt », und darum der « Wunsch nach Gleichheit, nach freien, offenen Beziehungen » (S. 160). 

Kategorie des «Absurden» aus? Logischerweise ja, doch nicht der menschlichen Erfahrung nach. Von letzterer handelt die vorliegende Erzählung eben auch. Von jenem Freund insbesondere, dem das Recht auf Glück willkürlich, durch staatliche Gewalt, entzogen wurde. Diese Willkür ist eine Form des Absurden im geschichtlichen Prozeß. Ihre Existenz läßt sich nicht leugnen, offen und vermummt tritt sie in stets neuer Gestalt auf. Wie ein Schatten, ein Todesschatten, begleitet das Unglück des Absurden, das Benno Ohnesorg erlitt, das Nachdenken des Erzählers, sein Schreiben und periodisch auch sein Leben. Gewiß bezeugt er die Möglichkeit des Glücks, das auch das Glück der anderen bedeutet, wenn er eine langwährende Liebesbeziehung offenlegt, die auf der Wechselseitigkeit der freien Hingabe, der Zuwendung, dem Schenken beruht (S. 171f.). Und doch ist die Erfahrung unhintergehbar, daß von einem Glück dieser Art der ihm nahestehende Freund für immer ausgeschlossen wurde : jener Absurdität wegen, die ein Ingrediens der Geschichte ist, ihre unverscheuchbare Mitgift. Allenfalls das Schreiben, die Kunst der Erinnerung, sichert dem früh Gestorbenen ein Überleben, das seiner würdig ist.

Dergestalt konvergieren in Timms Erzählung Geschichtsentwürfe deutscher und französischer Provenienz : der unaufgebbare Anspruch auf ein allgemeines «Glück im Diesseits », und der Einspruch des Absurden, der «alles Leid der Welt » auf sich zieht. Die Wegkreuzung, wo das eine und das andere, Anspruch und Einspruch, aufeinandertreffen, ist der Ort, an dem sich der "Mensch in der Revolte" bewähren muß.

Wie nur wenige Werke der Gegenwart versetzt Uwe Timms Erzählung unsere geschichtliche Existenz auf den Prüfstand. Mit unserer geschichtlichen durchleuchtet sie wiederholt auch unsere psychische Grundsituation. Die Art und Weise, wie der IchErzähler beispielsweise das philosophische Postulat der Unabhängigkeit auf das Motiv des Selbstschutzes hin transparent macht, wie er im Selbstschutz kontrapunktisch den gleichzeitigen Wunsch nach «Nähe, Selbstaufgabe und Hingabe " aufdeckt (S. 98 u. S. 100), ist ein Zeugnis luzider Seelenkunde. So skizziert Der Freund und der Fremde unter anderem auch Grundzüge einer modernen Bewußtseins- und Seelengeschichte.

\section{NOTES}

1. Zitiert nach Uwe тімм : Der Freund und der Fremde. Eine Erzählung, Köln, Kiepenheuer \& Witsch, 2005. 


\section{RÉSUMÉS}

Der folgende Beitrag zeigt, inwiefern Uwe Timms Text um einen Freund des Ich-Erzählers kreist - den während der Studentenunruhen 1967 erschossenen Benno Ohnesorg. Zugleich gerät damit die bewegte Zeit der Protestbewegung, aber auch ihre Vor- und ihre Nachgeschichte ins Blickfeld. Im Zusammenhang damit entwirft der Ich-Erzähler auch ein Porträt von sich selbst. Das die beiden Freunde während ihrer Vorbereitung auf das Abitur verbindende Ziel war ursprünglich der Beruf des Schriftstellers. Dieses Ziel konstituierte das «Anderswo» ihrer geheimen Wünsche und Sehnsüchte, jenes Anderswo, das ihnen während des Studiums zu entgleiten drohte. Der Beitrag erhellt die zentralen Impulse aus französischer Literatur und Philosophie, die beide Freunde empfingen, und er diskutiert das bedrängende Problem, ob dem Tod Benno Ohnesorgs, der für die Intensivierung der Protestbewegung von entscheidender Bedeutung war, der Status eines sinnvollen geschichtlichen Opfers zugesprochen werden kann. Als der authentische Erinnerungsort Ohnesorgs erscheint die Gedenkschrift des Erzählers, das spät erreichte "Anderswo".

L'article suivant montre comment le texte d'Uwe Timm parle d'un ami du narrateur, à savoir de Benno Ohnesorg, abattu par la police lors des émeutes étudiantes de 1967. En même temps, c'est toute l'époque agitée du mouvement protestataire, avec ses antécédents et ses conséquences, qui sont décrites. De même, le narrateur brosse un portrait de lui-même. Un même but liait à l'origine les deux amis pendant la préparation de leur baccalauréat : écrire. Ce but représente «l'ailleurs» de leurs souhaits et de leurs ambitions secrets, cet ailleurs qui menace de leur échapper au cours de leurs études universitaires. L'article éclaire les pulsions essentielles reçues en littérature et en philosophie françaises par les deux amis et il pose l'importante question suivante : la mort de Benno Ohnesorg, qui joue un rôle décisif dans l'escalade du mouvement protestataire, peut-elle avoir le sens d'un sacrifice historique? Le récit du souvenir du narrateur apparaît comme le lieu de mémoire authentique, l'ailleurs finalement atteint.

The following article shows how Uwe Timm's text speaks of a friend of the narrator, that is to say Benno Ohnesorg, shot by the police during the student riots of 1967. In the same time, it is all the turbulent time of the protesting movement, with its antecedents and its consequences that are described. Likewise, the narrator paints a picture of himself. A same goal linked originally the two friends during the preparation of their A-levels exam: the writing. This goal represents "the distance" of their secret wishes and their ambitions, this elsewhere that threats to escape them during their academic studies. The article puts the light on the essential pulses received in French literature and philosophy by both friends and he asks the following important question: Benno Ohnesorg's death, that plays a decisive role in the escalation of the protesting movement, may it have the sense of a historical sacrifice? The narrative of the narrator's memory appears as the authentic memory place, the elsewhere that is definitely reached.

\section{INDEX}

Mots-clés : Benno Ohnesorg

oeuvrecitee Der Freund und der Fremde, L'ami et l'étranger 
AUTEURS

GERT SAUTERMEISTER

Universität Bremen 